\title{
Contacts et emprunts entre discours : l'exemple du discours politique chez Deleuze
}

\author{
Sarah Leroy \\ UMR 7114 Modyco (CNRS/Université Paris Ouest Nanterre La Défense) \\ sarah.leroy@u-paris10.fr \\ Sylvain Loiseau \\ UMR 7114 Modyco (CNRS/Université Paris Ouest Nanterre La Défense) \\ sylvain.loiseau@u-paris10.fr
}

Les formes de contact entre discours peuvent être diverses : de l'utilisation de lexèmes spécialisés à la citation, voire l'allusion, en passant par des phraséologies ou des tours typiques. La notion $\mathrm{d}^{\prime}$ hétérogénéité énonciative ${ }^{1}$ permet d'analyser ces phénomènes en termes de double énonciation : un énoncé enchâssant fait entendre un énoncé enchâssé en le signalant par «un ensemble de marques linguistiques extrêmement hétérogènes » (Bres 2005 : 12). Il existe cependant des cas d'hétérogénéité qui semblent se présenter en discours de façon non marquée (par exemple, le détournement, l'allusion ou la citation allusive, comme dans l'exemple $1^{2}$ ) :

Ex 1. Juppé [surtitre]

Droit dans son box [titre]

L'affaire des emplois fictifs de la ville de Paris conduit l'ex-président du RPR au tribunal, huit ans après le début de l'enquête. [sous-titre]

(Libération, 29/09/03)

Un exemple comme celui-ci, malgré l'absence d'indices autres que le « léger trouble d'un " insolite " " (Authier-Revuz 2000: 218), se prête pourtant à une description en termes de double énonciation: l'énoncé droit dans son box enchâsse un autre énoncé (Je reste) droit dans mes bottes, antérieurement tenu par Alain Juppé, nommément cité dans le cotexte immédiat. Mais que dire des cas où ce qui est cité, repris, n'est pas un énoncé à proprement parler, mais une structure, un patron syntaxico-lexical, comme celui-ci, forme canonique du slogan revendicatif (exemple 2)?

Ex 2. Un pastis oui, un pastiche non! Un Ricard, un vrai !

(Publicité, 2006)

On le voit, plusieurs niveaux posent problème : d'une part, dans certains cas, le discours cité et détourné n'est pas nécessairement à rapporter à un énonciateur donné ; d'autre part, dans certains cas également, ce qui est cité est tellement ténu qu'on peut se demander s'il s'agit encore d'un énoncé ou plutôt d'une marque, d'un style.

Si on reprend cet exemple, pour lequel la notion d'hétérogénéité énonciative paraît donc devoir être complétée, on peut avancer que ce qui est enchâssé et qui peut être identifié, ce n'est pas un autre énonciateur mais un autre « registre discursif », en l'occurrence le registre politique, repris et évoqué dans un contexte publicitaire. De la même manière qu'un énoncé peut en enchâsser un autre, c'est ici un registre discursif qui en enchâsse un autre. Il paraît donc nécessaire de passer de la question de l'hétérogénéité des énonciateurs au fait de la pluralité des discours. Ceci implique de dissocier, au moins pour décrire ce type d'hétérogénéité, la question de l'hétérogénéité discursive des cadres polyphoniques 
et énonciatifs, ou encore d'envisager une hétérogénéité qui ne se situerait pas dans ces seuls cadres énonciatifs, mais qui s'inscrirait également au niveau des registres discursif.

Dans la suite de ce travail, nous désignerons donc par « discours » non pas un énoncé, production attestée ou attestable d'un énonciateur « réel » (fût-il fictif), mais des propriétés communes à des énoncés attestés, qui partagent à la fois des propriétés linguistiques et des contextes sociaux ou praxéologiques ${ }^{3}$. Dans cette acception, le terme discours est à rapprocher de celui de registre, de norme ou de genre mais il rend compte de catégories plus larges (champ littéraire ou presse $v s$ article, roman). Ainsi, cette catégorie de « discours » permet de distinguer, par exemple, discours publicitaire et discours politique, discours journalistique et discours littéraire, etc.

C'est dans cette optique que nous proposons de considérer les relations entre différents discours comme des contacts, comparables à des contacts interlinguistiques, et susceptibles, comme tous les phénomènes de contact, de donner lieu à des emprunts, sur le plan lexical bien sûr, mais aussi sur d'autres plans.

Après avoir précisé cette conception du discours et montré en quoi les phénomènes de contact, voire d'emprunt, qu'elle permet de décrire, complètent et prolongent des approches fondées sur l'analyse de phénomènes énonciatifs, de circulations des discours, de procédés métaphoriques, nous illustrerons l'intérêt de la démarche pour le discours philosophique en présentant quelques analyses de la présence du discours politique dans les textes du philosophe Deleuze.

\section{Discours, contacts et emprunts}

\subsection{Relations entre discours}

La conscience de la diversité des discours semble bien être un paramètre de toute activité langagière, comme l'atteste l'exemple 2, qui joue sur la mise en interaction de deux discours pour produire un dispositif dialogique. L'adoption d'une forme du discours politique (contestataire) y fait que l'énoncé du publicitaire se présente comme l'énoncé du consommateur et prétend coïncider exactement avec ses revendications, et non pas avec l'intérêt économique du commanditaire de la campagne publicitaire. Il y a donc représentation d'une identité d'intérêt entre le commanditaire et le consommateur, qui permettrait au premier de parler pour le second et de faire d'une publicité un dazibao.

Ce dispositif publicitaire a pour caractéristique majeure de construire, à travers l'invocation d'un autre discours, les pôles énonciatifs, par la reproduction du dispositif énonciatif de cet autre discours. Le slogan lui-même, dans le type de discours dont il relève, ne laisse « disponible » que la position de l'énonciateur, position que le consommateur est donc invité à prendre, et à partager avec l'annonceur. L'énonciataire du message publicitaire y est donc, en même temps, inclus, puisqu'il devient énonciateur de l'énoncé représenté. À la différence de ce qui se passe dans un énoncé dialogique «classique », cette répartition des pôles de l'énonciation n'est pas relative à des énonciateurs « réels 》 (ou homologues d'énonciateurs réels), mais bien construite, par l'utilisation du discours protestataire, via l'un de ses slogans les plus représentatifs. En effet, si le lien établi dans cet exemple entre le discours publicitaire et le discours politique fonctionne de façon aussi efficace, c'est aussi en raison de la fréquence des mises en relations entre ces deux discours. Ces relations sont elles-mêmes stabilisées et font donc partie des caractéristiques même des discours : c'est une propriété du discours publicitaire contemporain de pouvoir faire allusion au discours politique de façon privilégiée. De même, les connexions entre domaine politique et domaine sportif sont bien connues, en particulier à travers les métaphores; on voit ici que ces connexions se trouvent aussi exploitées dans des configurations dialogiques.

Le point de vue qu'on vient d'exposer, au lieu de faire dépendre l'identification et le décodage d'un dispositif énonciatif complexe d'un stock de connaissances purement encyclopédique, voire anecdotique, 
qui échapperait la description linguistique, pose que l'interprétation dépend, en partie, de compétences linguistiques des locuteurs, compétences relevant de la connaissance des discours et de leurs différences, de l'identification des normes discursives. C'est donc un tout pan de la compétence linguistique, fortement essentiel à la production comme à l'interprétation, qui peut ainsi être pris en compte.

Si l'on passe de l'énoncé « réel» au discours, comme nous venons de le faire, il est bien évident qu'il devient difficile de parler d'enchâssement énonciatif, notion qui implique une hiérarchisation dont l'énonciateur est le pivot. On associera donc au niveau énonciatif un niveau discursif, mettant en jeu d'autres phénomènes de citation et de reprise, complémentaires de ceux qu'une analyse des relations (co)énonciatives permet de faire apparaître. Pour le niveau discursif, on parlera plutôt de relation(s) $a ̀$ un discours, ou entre discours, ce qui entraîne un changement de perspective permettant d'envisager un parallèle entre contacts linguistiques, d'une langue ou d'une variété à une autre ${ }^{4}$, et contacts discursifs, d'un discours à un autre.

\subsection{Contacts entre discours}

La description des contacts entre discours, au lieu de rendre compte des relations entre énoncés, par l'intermédiaire d'une infime part de l'énonciation (c'est-à-dire l'énonciateur, pour l'essentiel), permet de considérer ces énoncés dans leur totalité, en intégrant l'ensemble de leurs propriétés (dialogiques, mais aussi lexicales, distributionnelles et actancielles) que la notion de discours permet de prendre en compte.

Cette proposition permet tout d'abord de répondre à un problème d'ordre terminologique: les phénomènes qui nous intéressent se trouvent en effet désignés par différents termes, ni tout à fait adéquats ni tout à fait spécifiques. Par exemple, la citation peut aussi renvoyer à du discours rapporté, le cliché et même l'allusion relèvent davantage de la stylistique littéraire, le défigement concerne plutôt le niveau lexical et l'idée de détournement a une dimension polémique qui ne concerne pas toujours nos objets. Le terme de contact permet donc d'éviter ces connotations et de mieux catégoriser le phénomène. Il n'est cependant lui-même pas neutre, puisqu'il renvoie avant tout aux relations interlangues ou intervariétés. Toutefois, il permet de faire un parallèle entre la diversité des langues et la diversité des discours. Le choix du terme contact met l'accent sur le fait que la diversité des discours est fondamentale dans cette perspective d'analyse : un discours inclut des orientations dialogiques et argumentatives qui le distinguent d'un autre. Du fait de cette hétérogénéité, les discours interagissent entre eux.

Si le recours aux notions de relations entre discours, de contact, apparaît nécessaire, il reste cependant à préciser plus clairement leurs contours. Rappelons tout d'abord l'existence de relations privilégiées entre discours : comme il y a des contacts de langues, on peut rapporter certaines relations entre certains discours à des contacts entre discours. On distingue cependant deux grandes «classes » de discours : certains d'entre eux empruntent peu, ou de façon très marginale (c'est par exemple le cas des discours juridique, administratif, scientifique ${ }^{5}$ ), et sont de ce fait ceux dont l'identification en tant que discours est la plus consensuelle; d'autres au contraire empruntent très facilement, en particulier tout ceux qui ont une forte composante axiologique (comme les discours politique, publicitaire, journalistique, philosophique). Plus généralement, les discours «emprunteurs» sont ceux que les typologies structurales du récit du siècle dernier appelaient « mythiques », par opposition aux discours «techniques $»^{6}$. Ainsi, si le discours juridique emprunte remarquablement peu aux autres discours et se satisfait de façon autarcique de ses propres normes, souvent archaïques et formulaires, a contrario, les emprunts du discours politique à d'autres discours sont légion, comme en témoigne l'exemple $3:$ l'allocution politique de la candidate du Parti Communiste Français à l'élection présidentielle intègre un segment relevant clairement du conversationnel oral.

Ex 3. «Depuis 20 ans, les politiques libérales ont échoué. On continue ou on fait du neuf? », demande la communiste [Marie-Georges Buffet]. 
On peut aussi penser que le contact entre un discours et un autre suppose l'utilisation d'une forme stéréotypique du discours emprunté. Ainsi, ce que la publicité emprunte au discours politique, c'est la forme figée du slogan c'est-à-dire une image canonique de ce discours «source ». Les exemples sont nombreux, mais on prendra pour illustration le cas de Jean-Marie Le Pen lorsqu'il évoque la nécessité de « préférer l'original à la copie »; il emprunte au dispositif argumentatif de la publicité comparative, sur le mode du «toujours imité, jamais égalé », autre formule. Cette stéréotypie participe donc fortement au fonctionnement des contacts entre discours.

\subsection{Emprunts entre discours}

Les situations de contact interlangues ou intervariétés ont généralement pour conséquence des changements dans les langues ou variétés concernées: les bilinguismes, les alternances et mélanges codiques, peuvent entraîner toutes sortes de phénomènes d'emprunt, à différents niveaux (lexical bien sûr, mais aussi phonologique par exemple).

Or on sait que l'emprunt linguistique ${ }^{7}$, défini comme « une unité ou un trait linguistique qui existait précédemment dans un parler B (dit langue source) et que A ne possédait pas, [utilisé et intégré dans] un parler A » (Dubois et al. 1994 : 177), selon qu'on le considère comme un processus ou un résultat, n'aboutit pas aux mêmes catégories. Ce fait linguistique, dont la description reste malaisée, gagne à être considéré comme un continuum entre les «deux catégories [qu'on observe en synchronie]: les pérégrinismes ou xénismes, c'est-à-dire les mots sentis comme étrangers et en quelque sorte cités (les Fremdwörter des linguistes allemands) et les emprunts proprement dits ou mots tout à fait naturalisés (les Lehnwörter) » (Deroy 1956: 224). Dans ce continuum, le xénisme, peu ou pas intégré à la langue cible, reste un mot étranger, mentionné avec référence au code linguistique d'origine et aux réalités étrangères, tandis que l'emprunt correspond au pôle opposé, celui de l'intégration achevée. On peut distinguer du xénisme le pérégrinisme, à mi-chemin de l'intégration, qui « renvoie encore à la réalité étrangère, mais [dont] la connaissance [du] sens est supposée partagée par l'interlocuteur » (Dubois et al. 1994 : 512). Par ailleurs, le terme d'emprunt est également le terme générique qui recouvre l'ensemble du continuum.

On peut penser que l'emprunt interdiscours est de même sujet à l'assimilation de formes et propriétés, et que des emprunts totalement intégrés (par exemple la dimension religieuse du rassemblement dans nombre de discours politiques) deviennent constitutifs du discours qui les accueille et par là même non repérables en tant qu'emprunts ${ }^{8}$. Il importe donc de bien différencier les emprunts interdiscours «non acclimatés », l'équivalent de xénismes ou de pérégrinismes discursifs, introduisant un décalage qui demande à être identifié comme tel par l'énonciataire des emprunts adoptés. Seuls les premiers produisent un effet de citation, l'hétérogénéité textuelle ne pouvant être identifiée qu'en étant rapportée à l'hétérogénéité d'un discours. Ainsi, dans l'exemple 4, la formule Prière d'insérer, habituellement utilisée dans le cadre des relations entre éditeurs et presse (et par ailleurs elle-même suffisamment figée pour former une dénomination stable ${ }^{9}$ ) et construite sur le modèle des injonctions réglementaires prière de + inf. (Prière de ne pas déranger, Prière de noter, etc.), détonne au sein du discours journalistique, qui n'a pas pour habitude d'utiliser la modalité injonctive, en particulier dans un titre.

Ex 4. Françoise Bernon, 43 ans. Ancienne assistante sociale, elle dirige une imprimerie qui emploie les rejetés du système. [surtitre]

Prière d'insérer. [titre]

(Libération, 18/02/03)

Envisager ces phénomènes en terme d'emprunt (au sens général du terme) permet de dissocier la question du repérage de celle du décodage : il est en effet fréquent qu'un énonciataire, sans forcément pouvoir le 
rapporter à du connu, perçoive un décalage, sente qu' « il y a quelque chose » de décalé dans un discours, sans pouvoir dire quoi. Ce qui est alors perçu, c'est le caractère " étranger », discursivement parlant, du segment concerné, son extranéité vis-à-vis du discours dans lequel il est inséré. Cette première étape (qui n'est pas systématique et peut être ignorée par un locuteur peu au fait de telles ou telle norme discursive, évidemment éminemment culturelle) est distincte de l'étape du décodage, qui fait appel à des compétences linguistiques plus variées : compétence « discursive », certes, qui permet de rapporter le titre au mot imprimerie du chapeau résumant l'article, mais aussi lexicale (pour le décodage de l'isotopie rejetés - insérer par l'intermédiaire du terme réinsertion).

Par ailleurs, un élément « autre » qui ne relèverait pas d'un discours autre (des citations allusives au sein d'un même discours, littéraire par exemple) ne serait pas repéré par cet écart discursif, mais seulement décodé par un locuteur au fait de l'énoncé initial. C'est ce qu'on voit avec l'exemple 5, emprunté à Bres (in Détrie et al. 2001: 88) qui précise «Qui a lu Flaubert ne peut pas ne pas entendre sous la voix du narrateur de Je m'en vais $\left(\mathrm{E}_{1}\right)$, la voix à la fois cachée et exhibée du narrateur de L'Éducation sentimentale $\left(\mathrm{e}_{1}\right)$ ». Il faut en effet avoir lu Flaubert, faute de quoi la citation passe totalement inaperçue, aucun indice d'emprunt entre discours ne permettant de la détecter.

Ex 5. Il connait la mélancolie des restauroutes, les réveils acides des chambres d'hôtel pas encore chauffées, l'étourdissement des zones rurales et des chantiers, l'amertume des sympathies impossibles.

(Je m'en vais, J. Echenoz)

Ex 5.' Il connut la mélancolie des paquebots, les froids réveils sous la tente, l'étourdissement des paysages et des ruines, l'amertume des sympathies interrompues.

(L'Éducation sentimentale, G. Flaubert)

On peut donc distinguer entre des détournements intradiscours, qui relèvent du niveau énonciatif et des emprunts interdiscours, qui relèvent du niveau discursif.

\section{Illustration : l'emprunt au discours politique chez Deleuze}

\subsection{Les discours de Deleuze}

Si le discours philosophique est particulièrement intéressant pour une observation de phénomènes d'emprunts entre discours, c'est qu'il est particulièrement «emprunteur » (cf. supra). Nous proposons d'illustrer, dans un corpus constitué du dyptique Capitalisme et schizophrénie de G. Deleuze et F. Guattari, des phénomènes d'emprunts interdiscours et d'en proposer une rapide typologie. Ce dyptique regroupe deux textes représentatifs de la période avant-gardiste de Deleuze : l'Anti-Edipe (désormais $A O$ ) et Mille Plateaux (désormais $M P$ ).

Une analyse globale de l'œuvre de Deleuze, au moyen d'analyses factorielles, permet de proposer une périodisation du corpus. Une première période se dégage, regroupant les textes « de jeunesse » (environ un tiers de l'œuvre globale), qui possèdent toutes les marques du discours académique; dans une seconde période, ces marques d'académisme s'estompent derrière d'autres, peu attendues dans le discours philosophique, qui font apparaitre une "coloration » politique d'abord, puis littéraire dans les derniers textes de l'œuvre. Cette périodisation s'appuie sur l'analyse des ponctèmes, des temps et des modes, des personnes, de traits grammaticaux comme l'indéfini et le nombre (sur toutes les parties du discours) et de propriétés typographiques (notes, titraille, guillemets, italiques...) ${ }^{10}$. La répartition des variables significatives sur l'axe diachronique permet finalement de caractériser trois périodes : dans la première, celle des textes « de jeunesse », le nous d'auteur, le point-virgule, le subjonctif, les italiques et les titres 
prévalent; dans une deuxième, c'est le vous et le $t u$, le point d'exclamation, l'impératif et le futur qui s'imposent; une troisième, enfin, se signale par le on et le trait indéfini, les points de suspension, l'imparfait. Ces indices nous permettent d'avancer que la deuxième partie témoigne de la " politisation » du discours philosophique de Deleuze dans les années 1968-1980, tandis que la troisième témoigne d'un repli vers le discours littéraire. C'est sur la base de cette périodisation globale qu'on s'intéresse aux influences entre discours chez Deleuze, et plus particulièrement aux modalités de l'influence du discours politique, dans les deux textes principaux de la deuxième période.

Les phénomènes d'emprunt dans ce corpus doivent être évidemment rapportés à certaines caractéristiques de ce corpus et de son contexte. D'une part, la position éminente acquise par les sciences humaines dans la configuration des discours autour des années soixante se traduit par une importation massive de son vocabulaire dans le discours philosophique. On peut d'ailleurs faire l'hypothèse que c'est une caractéristique du discours philosophique que d'être particulièrement sensible aux discours intellectuellement dominants d'une époque (voir par exemple Rastier 2001b sur l'influence du discours scientifique sur les « idéologues», ou Cassin 1998 sur le fait que l'un des premiers textes philosophiques, le Poème de Parménide, est un démarquage de l'Odyssée). D'autre part, Capitalisme et schizophrénie s'inscrit explicitement dans un programme de renouvellement et de subversion des formes du discours philosophique: " il [L'Anti-CEdipe] est encore bien universitaire, assez sage, et ce n'est pas la pop'philosophie ou la pop'analyse rêvées » (Deleuze 1990: 16). Ce programme de renouvellement emprunte notamment à une esthétique du collage et du fragment.

Pour ces deux principales raisons, ce corpus est particulièrement riche en phénomènes d'emprunt entre discours. Si de nombreux discours sont convoqués par Deleuze, s'exprimant dans le genre philosophique de l'essai (sciences humaines, psychanalyse, médicale), l'influence du discours politique est dominante, et c'est donc sur celle-ci que nous nous arrêtons. Les paragraphes ci-dessous font le point sur les phénomènes d'emprunt au discours politique, emprunts qu'on rapporte à trois grands ensembles: le lexique, la phraséologie, et l'éthos.

\subsection{Les emprunts interdiscours chez Deleuze}

\subsubsection{Emprunts lexicaux}

Le niveau lexical est, traditionnellement, celui qui fait le mieux apparaître les phénomènes d'emprunt. L'analyse d'un terme comme fascisme, éminemment politique du point de vue sémantique (ce substantif désigne une doctrine politique caractérisée par la toute puissance de l'État) comme du point de vue historique (il reste lié à la période mussolinienne) et axiologique (il est toujours fortement connoté négativement), témoigne de la reconfiguration d'un emprunt dans son discours d'accueil, et de l'exploitation sémantique et expressive qui peut en être faite.

Ex 6. Le « cinq heures du soir » de Lorca, quand l'amour tombe et le fascisme

se lève.

$(M P: 319)$

Ex 7. La musique a soif de destruction, tous les genres de destruction, extinction, cassage, dislocation. N'est-ce pas son « fascisme » potentiel?

(MP:367-368)

Dans ces deux exemples, la valeur strictement axiologique de fascisme est prédominante, au-delà même de celle qui lui appartient dans son discours d'origine. C'est cette valeur qui passe au premier plan, le sens lui-même étant modifié par l'opération d'emprunt (ainsi, l'antonyme de fascisme dans le corpus est révolutionnaire, et non une lexie désignant une doctrine opposée). L'emprunt entre discours exploite ici, 
de façon quasi-exclusive et donc fortement développée, une des dimensions du mot dans son discours originel.

L'exemple 6 illustre cette radicalisation, par la lexicalisation d'oppositions axiologiques : il y a en effet une opposition entre les traits sémantiques inhérents /vers le haut/ et/vers le bas/ des sémèmes de tomber et monter, qui appartiennent à la même classe lexicale ${ }^{11}$. Ceci implique une opposition antinomique afférente entre les sémèmes d'amour et de fascisme, dont l'opposition sur le plan moral est représentée comme aussi exclusive que l'opposition entre le haut et le bas sur le plan physique. Cette opposition est rapportée, via « cinq heures du soir », au topos classique, voire littéraire, de l'opposition du jour et de la nuit. De plus, il y a une contradiction entre les plans physiques et moraux : amour, qui occupe le pôle positif sur le plan moral, est affecté d'une direction /vers le bas/, c'est-à-dire conventionnellement du pôle négatif sur le plan physique. On retrouve dans ce tableau, littéralement, d'un crépuscule, la «vision crépusculaire du monde » que décrit Angenot (1982) dans le genre pamphlétaire : le monde est affecté d'un renversement généralisé des valeurs. Cet exemple montre le caractère absolu des oppositions, drainé par l'exploitation purement axiologique d'un terme politique: les positions axiologiques sont maximalisées, seuls comptent les pôles extrêmes.

Ce qui se joue avec cet emprunt, outre la coloration politique d'un texte militant du discours philosophique et une utilisation évaluative du terme emprunté, c'est la représentation même du discours politique dans le discours philosophique : l'emprunt est le corollaire de l'expression d'une image - ici particulièrement stéréotypée - du discours source dans le discours d'accueil : le discours politique est donné à voir comme un "réservoir d'axiologie», comme un lieu structuré par des oppositions axiologiques.

L'emploi exclusivement évaluatif de cette lexie issue du domaine politique a pour conséquence sur le plan sémantique que, n'étant plus rattachée à son domaine privilégié (le politique), elle devient compatible avec n'importe quel autre domaine. C'est ce que montre l'exemple 7, où la lexie fasciste est ainsi mobilisée dans le domaine musical - les guillemets témoignant cependant de l'écart assumé avec son acception « originale». L'emprunt lexical au discours politique contribue ainsi à la connexion de domaines à travers une échelle évaluative partagée.

\subsubsection{Les emprunts phraséologiques}

L'emprunt interdiscours ne se limite cependant pas au plan lexical, mais intéresse des formes aux contours plus lâches, relevant de la phraséologie ou de la formule. L'Anti-Edipe puis, plus encore, Mille Plateaux, en offrent de nombreux exemples. Ces exemples relèvent du «collage» : des slogans, des phraséologies, issus d'une tradition discursive politique et militante, sont directement intégrés dans le discours philosophique. Ces collages laissent diversement paraître les marques de leur suture. Ainsi, l'exemple 8 prend encore la forme typographique d'une citation (« La police avec nous! »), tandis que les exemples 9 (où les deux points ouvrent une séquence de slogans) et, plus encore, 10 (où le slogan «phallus et castration oui, (Edipe non!» est totalement intégré dans le flux discursif, au prix d'une anacoluthe) ne distinguent plus ces différents éléments hétérogènes; la forme «collée » est transposée telle quelle.

Ex 8. Tout d'un coup, c'est comme si le corps des notaires avançait en Arabes ou en Indiens, et puis se reprenait, se réorganisait : un opéra-comique, dont on ne sait pas ce qui va sortir (il arrive même qu'on crie : « La police avec nous ! »)

$(M P: 454)$

Ex 9. Écrire à $\mathrm{n}, \mathrm{n}-1$, écrire par slogans : Faites rhizome et pas racine, ne plantez jamais! Ne semez pas, piquez! Ne soyez pas un ni multiple, soyez des multiplicités! Faites la ligne et jamais le point! La vitesse transforme le point en 
ligne! Soyez rapide, même sur place! Ligne de chance, ligne de hanche, ligne de fuite. Ne suscitez pas un Général en vous !

$(M P: 36)$

Ex 10. Et n'est-ce pas là toujours la force de Lacan, avoir sauvé la psychanalyse de l'œdipianisation forcenée à laquelle elle liait son destin, [...] de le réinterpréter à partir de cet appareil, la loi et le signifiant, phallus et castration oui, Edipe non !, l'âge despotique de l'inconscient.

$(A O: 257)$

Remarquons que l'exemple 10 met en scène le même modèle de slogan stéréotypique ( $\mathrm{X}$, oui !, Y, non !») que celui observé supra dans le discours publicitaire (exemple 2).

Ces emprunts privilégient tous une forme particulière, voire un type de textes, celui du slogan, dont la syntaxe prend le contre-pied des conventions du discours philosophique: les phrases sont courtes, souvent nominales; le système des pronoms personnels est pratiquement réduit aux premières et deuxièmes personnes, qui sont presque exclues du discours philosophique académique; l'impératif domine dans le système verbal, alors que, là encore, c'est un temps par excellence proscrit dans le discours philosophique. L'opposition porte également sur les registres et sur la modalité : ces collages miment un oral spontané prototypique, y compris dans le jeu de mot (paronomase fondée sur une métathèse hanches/chance de l'exemple 9) et de la créativité néologique (" faire rhizome », etc., de l'exemple 9 également).

Certains passages permettent d'observer plus finement le fonctionnement et la rentabilité de tels emprunts phraséologiques.

Ex. 11. Or c'est toujours du fond de son impuissance que chaque centre de pouvoir tire sa puissance : d'où sa méchanceté radicale, et sa vanité. Plutôt être un minuscule quantum de flux qu'un convertisseur, un oscillateur, un distributeur molaire ! Pour en revenir à l'exemple monétaire [...]

(MP, pp. 276-277)

Ex. 12. À l'ensemble des strates, le CsO oppose la désarticulation (ou les $\mathrm{n}$ articulations) comme propriété du plan de consistance, l'expérimentation comme opération sur ce plan (pas de signifiant, n'interprétez jamais !), le nomadisme comme mouvement (même sur place, bougez, ne cessez pas de bouger, voyage immobile, désubjectivation).

(MP : 197-198)

Dans les exemples 11 et 12 , deux discours sont mis en parallèle : les formulations empruntant à la phraséologie militante (sur le modèle du mot d'ordre) redoublent dans une certaine mesure, sur un registre politique, ce qui a été précédemment formulé en termes philosophiques «classiques ». Ils se présentent donc comme une reformulation, voire une traduction, d'un discours dans l'autre. Dans l'exemple 12, le parallélisme des deux développements est systématisé par l'usage des parenthèses. À deux reprises, une formulation "philosophique» essentiellement nominale (substantifs construits abstraits d'action, tous déverbaux y compris le néologisme nomadisme, construit sur une règle morphologique déverbale) précède une formulation «politique » verbale (impératif, seconde personne). D'un côté l'abstraction universalisante, de l'autre l'injonction militante à agir.

Enfin, ce parallélisme peut s'étendre au niveau du contenu topique. L'exemple 11 contient ainsi un topos bien établi dans chacun des discours. Dans la formulation "philosophique », on retrouve la figure de la dialectique philosophique (et sa dimension paradoxale) : des propriétés contradictoires sont affectées tour 
à tour à un même objet, à mesure qu'est représenté le dévoilement de la connaissance : l'impuissance est, au fond, source de puissance. Dans la formulation "politique», on lit également un topos du renversement, celui qui fait préférer une situation « minoritaire » à une situation de force.

La dimension phraséologique de l'emprunt au discours politique chez Deleuze, exposée ci-dessus, se réalise à travers l'aménagement de parallélismes, de mises en écho, qui permet une lecture sur les deux plans: ces phraséologies se trouvent essentiellement dans des contextes d'équivalence avec des formulations plus classiquement philosophiques. On peut donc se poser la question de leur autonomie, et donc de leur intégration ou, au contraire, de leur caractère " xénique », au sein du discours d'accueil.

\title{
2.2.3 L'emprunt d'un éthos politique
}

Au-delà du lexical et du phraséologique, toujours identifiable sur la base de marques formelles, c'est le système actanciel du texte, et la répartition des actants entre différents univers de valeur, qui semble, en dernière analyse, relever d'une influence du discours politique. Comme dans le discours politique en effet, le locuteur est représenté à travers un actant collectif (dont les descriptions du discours politiques ont mis en évidence l'importance) et il partage le même univers de valeur que son destinataire. Le discours contribue à la création d'une identité collective et à l'affirmation ou l'imposition d'un objectif commun à travers un devoir-être commun. Dans les textes de Deleuze, la construction du locuteur comme actant, ses rôles et ses évaluations axiologiques semblent directement influencés par le discours politique.

Ce niveau d'emprunt peut néanmoins se manifester via des indices linguistiques tels que l'emploi d'un pronom personnel : dans le discours politique, la construction d'une identité collective passe typiquement par l'emploi du pronom nous. Ainsi l'étudiant dans le discours syndical, Habert et Lefevre (1985) soulignent que « [1]'emploi qui est fait du nous aboutit non pas à distinguer les différentes voix, mais à les fondre», et s'interrogent sur « le rôle des phrases en nous dans la création d'une identité collective ». De même, Mothé (1973) indique :

Les militants, même lorsqu'ils sont extrêmement minoritaires, ne parlent presque jamais en leur nom personnel, ils préfèrent de loin employer le nous montrant qu'ils ne font qu'exprimer le sentiment, les idées, les opinions de la classe tout entière qu'ils prétendent à chaque instant représenter.

On retrouve chez Deleuze l'utilisation de ce pronom personnel de deuxième personne, avec précisément cette valeur de création d'une identité collective, comme on le voit dans l'exemple 13.

\begin{abstract}
Ex 13. Nous sommes à l'âge des objets partiels, des briques et des restes. Nous ne croyons plus en ces faux fragments qui, tels les morceaux de la statue antique, attendent d'être complétés et recollés pour composer une unité qui est aussi bien l'unité d'origine. Nous ne croyons plus à une totalité originelle ni à une totalité de destination. Nous ne croyons plus à la grisaille d'une fade dialectique évolutive, qui prétend pacifier les morceaux parce qu'elle en arrondit les bords. Nous ne croyons à des totalités qu'à côté. Et si nous rencontrons une telle totalité à côté de parties, c'est un tout de ces parties-là, mais qui ne les totalise pas, une unité de toutes ces parties-là, mais qui ne les unifie pas, et qui s'ajoute à elles comme une nouvelle partie composée à part.
\end{abstract}

$(A O: 50-51)$

Dans cet exemple, le locuteur s'inscrit dans un actant collectif qui est consolidé par une intense dramatisation. À l'annonce prophétique («nous sommes à l'âge des [...]»), succède la posture démystificatrice (« Nous ne croyons plus $[\ldots] »)$, renforcée ensuite par une dénonciation de la situation présente (« la grisaille d'une fade dialectique évolutive »); la période se clôt, après cet intense recours au 
pathétique, par l'énoncé d'une « solution » dans des phrases positives mais encore affectées de restriction et de condition : «nous ne croyons qu'à [...]. Et si nous [...], c'est [...]»

À un autre niveau, non repérable formellement celui-là, celui de la structure narrative, c'est l'installation d'un univers axiologique binaire, dans lequel la définition des univers de valeur est particulièrement importante pour la définition des actants : ces actants, ou au moins leur extension, sont constitués à travers leur participation à un même univers de valeur. Le partage d'une finalité et de valeurs entre locuteur et destinataire représentés est certes traditionnel dans le discours philosophique, mais il exclut maintenant les valeurs de neutralité de d'objectivité pour se fonder sur un "devoir» extérieur à la pure quête d'un «savoir ». Par exemple, comparant les textes politiques et les textes philosophiques de Jaurès, Provost (1969: 51-52) note de même que :

\section{[...] l'étude des deux types d'énoncés analysés (philosophique et politique) met en évidence un phénomène de passage de l'énoncé considéré comme "non engagé », « objectif», à l'énoncé défini comme politique et «non scientifique ».}

Pour caractériser ce passage, elle souligne l'importance de la modalisation dans le discours politique et à travers elle l'établissement d'un actant collectif où « [...] le locuteur (Jaurès) ne s'exclut pas de l'actant ».

Chez Deleuze, on retrouve cette orientation axiologique : l'actant représenté par le nous de l'exemple 14 est défini de façon différentielle, par opposition au pôle des antivaleurs.

Ex 14. [...] surveiller jusqu'en nous le fasciste, et aussi le suicidaire et le dément $[\ldots]$

$(M P: 204)$

De plus, d'une manière qui rappelle également discours politique, la légitimité du locuteur à valoir pour la collectivité est garantie dans cet exemple par une surenchère dans l'adéquation aux valeurs collectives, qui se traduit par une radicalisation des oppositions et des dénonciations, allant jusqu'à l'« autocritique ».

La stratégie persuasive semble donc reposer, en partie, sur l'invocation d'un devoir commun auquel le locuteur se représente comme soumis, ce qui fait de son énoncé une simple expression de cet univers de valeur: le destinataire est mis dans l'alternative d'accepter la vérité du propos ou de trahir les idéaux invoqués.

\section{Conclusion}

Pour rendre compte de phénomènes subtils semblant dévoiler la présence de discours «autres » au sein d'un discours donné, dont les approches énonciatives peinent à rendre compte car, justement, ils se situent à un niveau qui n'est pas celui de l'énonciation, nous nous sommes appuyés sur la notion de discours comme ensemble d'énoncés ayant en commun des propriétés linguistiques et des contextes sociaux ou praxéologiques.

Sur la base de cette conception du discours, nous avons développé un parallèle entre (variétés de) langues et discours, permettant, selon nous, de décrire les phénomènes concernés comme résultant de contacts entre discours, et pouvant alors être considérés comme des emprunts internes ${ }^{12}$, des emprunts discursifs plus ou moins intégrés selon les cas.

L'examen du corpus deleuzien, ou plutôt d'une partie de ce corpus sélectionnée sur la base d'indices permettant d'y supposer une forte influence du discours politique, a en effet révélé, marqués par des emprunts extrêmement variés, des phénomènes de de contact entre discours philosophique et discours politique. Les emprunts mis en lumière et analysés concernent aussi bien le lexique, avec l'importation de termes spécifiquement politiques, que des formules comme le slogan, moins aisément repérables, plus 
hétérogènes, et pourtant fortement exploitées dans un parallèle entre philosophique et politique, ou que des configurations dialogiques et actancielles exhibant un ethos. À chaque fois, le discours politique, et notamment ses valeurs axiologiques, est représenté dans le discours d'accueil.

Enfin, outre leur capacité descriptive, ces situations de contact entre discours nous semblent particulièrement intéressantes pour une perspective typologique : plutôt que de faire dépendre l'objectif d'une typologie des discours de critères a priori, elles permettent d'observer des disjonctions et des rapprochement effectifs entre discours.

\section{Références bibliographiques}

Angenot, M. (1982). La Parole pamphlétaire. Typologie des discours modernes. Paris : Payot.

Authier-Revuz, J. (1984). Hétérogénéité(s) énonciative(s). Langages, 73, pp. 98-111.

Authier-Revuz, J. (2000). Aux risques de l'allusion. In Murat, M. (éd.), L'Allusion dans la littérature. Actes du XXIV Congrès de la Società Universitaria per gli Studi di Lingua e Letteratura Francese (SUSLLF). En Sorbonne, novembre 1998. Paris : Presses de l'Université de Paris-Sorbonne, pp. 209-235.

Bakhtine, M. (1979/1984). Pour une esthétique de la création verbale. Paris : Gallimard.

Bres, J. (1999a). Entendre des voix: de quelques marqueurs dialogiques en français. In Bres, J., DelamotteLegrand, R., Madray-Lesigne, F., Siblot, P. (éd.), L'Autre en discours, Montpellier : Publications Montpellier 3, pp. 191-212.

Bres, J. (1999b). Vous les entendez ? Analyse du discours et dialogisme. Modèles linguistiques, 20-2, pp. 71-86.

Bres, J. (2005). Sous la surface textuelle, la profondeur énonciative. Ébauche de description des façons dont se signifie le dialogisme de l'énoncé. In Haillet, P.P. \& Karmaoui, G. (éd.), Regards sur l'héritage de Mikhaïl Bakhtine, Cergy-Pontoise : Université de Cergy-Pontoise, pp. 11-33.

Cassin B. (1998) «Quand lire c'est faire », introduction à Parménide, Sur la nature ou sur l'étant. Paris : Seuil.

Deleuze, G. (1990). Pourparlers. Paris : Minuit.

Deroy, L. (1956). L'Emprunt linguistique. Paris : Les Belles Lettres.

Détrie, C., Siblot, P., Verine, B. (éd.). (2001). Termes et Concepts pour l'analyse du discours. Une approche praxématique. Paris : Champion.

Dubois, J., Guespin, L., Giacomo, M., Marcellesi, C., Marcellesi, J.B., Mevel, J. (1994). Dictionnaire de linguistique et des sciences du langage. Paris : Larousse.

Gadet, F. \& Jones, M. (2008). Variation, Contact and Convergence in French Spoken Outside France. Journal of Language Contact THEMA, 2, pp. 238-248.

Habert, B. \& Lefevre, J. (1985). Nous chez Edmond Maire et Henri Krasucki. Mots, 11, pp. 191-221.

Leroy, S. (2005). Le détournement dans les titres de presse : un marquage dialogique ?. In Bres, J., Haillet, P.P., Mellet, S., Nølke, H., Rosier, L. (éd.), Dialogisme, polyphonie: approches linguistiques. Actes du colloque international de Cerisy-la-Salle, 2-9 septembre 2004, Bruxelles : De Boeck.Duculot, pp. 201-214.

Loiseau S. (2006) Sémantique du discours philosophique chez Deleuze : du corpus aux normes, Thèse de doctorat, Université Paris 10 Nanterre.

Loiseau S. (2003) «Philosophical discourse from autonomy to engagement: Deleuze commentator of Spinoza ». In Flottum K. \& Rastier F. (éd.), Academic discourses - Multidisciplinary Approaches, Oslo : Novus, pp. 36-54. 
Loiseau S. \& Rastier F. (à paraître) «La linguistique des textes philosophiques : du «structuralisme » à la linguistique des corpus numériques ». In Actes du colloque La philosophie dans le moment des années 60, École Normale Supérieure (Ulm), 17-18 octobre 2008.

Meillet, A. (1905-1906). Comment les mots changent de sens. L'Année sociologique, 9, pp. 1-38.

Mothé, D. (1973). Le Métier de militant. Paris : Seuil.

Provost, G. (1969). Approche du discours politique : «Socialisme » et « socialiste » chez Jaurès. Langages, 13, pp. 51-68.

Rastier, F. (2001a). Arts et Science du texte. Paris : Presses Universitaires de France.

Rastier, F. (2001b) «l'Être naquit dans le langage : un aspect de la mimésis philosophique », Méthodos, 1, pp. 103132.

Saussure, F. de (1915/1972) (Bally C. \& Séchehaye A. éd.). Cours de linguistique générale. Paris : Payot.

Todorov, T. (1981). Mikhaïl Bakhtine : le principe dialogique. Paris : Seuil.

${ }^{1}$ Cf. Authier-Revuz (1984), Bakhtine (1979), Bres (1999a et 1999b), Todorov (1981).

${ }^{2} \mathrm{Cf}$. Leroy (2005), dont est tiré cet exemple de titre de presse.

${ }^{3}$ Voir par exemple Rastier (2001a).

${ }^{4}$ « Language always evolves in a social context, which necessarily implies contact between speakers, ie between linguistic varieties if not between languages themselves. » (Gadet \& Jones 2008 : 245).

${ }^{5}$ Ceux que déjà Saussure (1972:41) qualifiait de « langues spéciales (langue juridique, terminologie scientifique, etc.) », et pour lesquels on parle maintenant de langues de spécialité.

${ }^{6}$ Distinction utilisée par exemple dans Rastier (2001b)

${ }^{7}$ Cf. Deroy (1956).

${ }^{8}$ L'intégration progressive des emprunts peut d'ailleurs déplacer les frontières entre discours.

9 « Encart imprimé contenant des indications sur un ouvrage et qui est joint aux exemplaires adressés à la critique » (Petit Robert 1990, s.v. insérer).

${ }^{10}$ D'autres éléments, tels que le changement d'éditeur (passage des Presses Universitaires de France aux éditions de Minuit, repaire des avant-gardes intellectuelles de l'époque), sont à prendre en compte.

${ }^{11}$ En effet, une opposition de sèmes n'est inhérente que si les deux termes de l'opposition appartiennent à la même classe lexicale.

${ }^{12}$ Cf. Meillet (1905-1906). 